\title{
Two-port versus three-port video-assisted thoracoscopic surgery for primary spontaneous pneumothorax: feasibility, postoperative outcome and long-term recurrence rates
}

Stephen Fung ${ }^{* \dagger}$, Hany Ashmawy ${ }^{\dagger}$, Sami Safi, Anja Schauer, Alexander Rehders, Levent Dizdar, Georg Fluegen ${ }^{\dagger}$ and Wolfram Trudo Knoefel ${ }^{\dagger}$

\begin{abstract}
Background: Two-port VATS (2-P-VATS) and three-port VATS (3-P-VATS) are well-established techniques for surgical therapy of primary spontaneous pneumothorax (PSP). However, comparisons of both techniques in terms of postoperative outcome and recurrence are limited.

Methods: From January 2010 to March 2020, we retrospectively reviewed data of 58 PSP patients who underwent VATS in our institution. For statistical analysis, categorical and continuous variables were compared by chi-square test or Fisher's exact test and the Student's t-test, respectively. Twenty-eight patients underwent 2-P-VATS and 30 were treated with 3-P-VATS. Operation time, length of hospital stay (LOS), total dose of analgesics per stay (opioids and non-opioids), duration of chest tube drainage, pleurectomy volume (PV), postoperative complications and recurrence rates were compared between both groups.

Results: Clinical and surgical characteristics including mean age, gender, Body-Mass-Index (BMI), pneumothorax size, smoking behaviour, history of contralateral pneumothorax, side of pneumothorax, pleurectomy volume and number of resected segments were similar in both groups. The mean operation time, LOS and total postoperative opioid and non-opioid dose was significantly higher in the 3-P-VATS group compared with the 2-P-VATS group. Despite not being statistically significant, duration of chest tube was longer in the 3-P-VATS group compared with the 2-P-VATS group. In terms of postoperative complications, the occurrence of hemothorax was significantly higher in the 3-P-VATS group (3-P-VATS vs. 2-P-VATS; $P=0.001$ ). During a median follow-up period of 61.6 months, there was no significant statistical difference in recurrence rates in both groups (2/28 (16.7\%) vs. 5/30 (7.1\%); $p=0.274)$.
\end{abstract}

Conclusion: Our data demonstrate that 2-P-VATS is safer and effective. It is associated with reduced length of hospital stay and decreased postoperative pain resulting in less analgesic use.

Keywords: Two-port VATS, Three-port VATS, Outcome, PSP

*Correspondence: stephen.fung@med.uni-duesseldorf.de

${ }^{\dagger}$ Stephen Fung, Hany Ashmawy, Georg Fluegen and Wolfram Trudo Knoefel contributed equally to this work

Department of Surgery, University Hospital Duesseldorf and HeinrichHeine-University Duesseldorf, Moorenstrasse 5, 40225 Duesseldorf, Germany

\section{Background}

As defined in the current German S3 guidelines, primary spontaneous pneumothorax (PSP) describes the presence of air without preceding trauma or underlying pulmonary disease within the pleural space of young patients under 45 years of age [1] The incidence of PSP 
has been reported with approximately $1-9.8$ and 7-24 cases per 100,000 individuals per year in females and males respectively $[2,3]$ Due to the low recurrence und morbidity rates, current guidelines $[1,4,5]$ recommend VATS for surgical treatment of PSP. In the last decades, thoracic surgery has evolved from thoracotomy to videoassisted thoracoscopic surgery (VATS). While three-port VATS (3-P-VATS) remains the gold standard for PSP treatment, recent publications have demonstrated the advantages of uniportal VATS in terms of postoperative pain and paraesthesia, analgesic use, length of hospital stay, cosmetic results and patient satisfaction scores in specialized settings [6-11]. However, only a few studies have analysed and compared the postoperative outcome of the widely implemented two-port VATS (2-P-VATS) with 3-P-VATS $[8,12]$. Therefore, the aim of this study was to evaluate and compare the outcome and long-term recurrence rates of PSP patients treated with 2-P-VATS and 3-P-VATS in our institution.

\section{Material and methods \\ Patients}

We retrospectively analysed data of 58 patients who underwent video-assisted thoracoscopic surgery (VATS) for primary spontaneous pneumothorax (PSP) in our institution between January 2010 and March 2020. Twenty-eight patients were treated using 2-P-VATS and 30 patients underwent the conventional 3-P-VATS. Indication for surgery was persistent air leak for more than 5 days after chest tube treatment on first episode (2-P-VATS: $\mathrm{N}=8$; 3-P-VATS: $\mathrm{N}=10$ ), second ipsilateral pneumothorax (2-P-VATS: $\mathrm{N}=12$; 3-P-VATS: $\mathrm{N}=15$ ), synchronous bilateral spontaneous pneumothorax (2-P-VATS: $\mathrm{N}=5$; 3-P-VATS: $\mathrm{N}=3$ ), and spontaneous hemopneumothorax (2-P-VATS: $\mathrm{N}=3$; 3-P-VATS: $\mathrm{N}=2$ ). Prior to surgery, all the patients received a CT (computer tomography) scan of the thorax to detect any bullous disease. For each patient, medical charts were reviewed to retrieve the following variables: age, sex, body mass index (BMI), side of pneumothorax, pneumothorax size, smoking behaviour, number of resected lung segments (if any), volume of resected parietal pleura, length of hospital stay (days), duration of chest drainage (days), total dose of opioid and non-opioid use per stay, operation time and postoperative complications (Tables 1 and 2). Only PSP patients with completed follow-up data were included in this study. All the patients underwent VATS with partial pleurectomy and bullectomy when blebs where evident. Patients who underwent other treatment modalities such as thoracotomy, apical pleurectomy or suffered a different pneumothorax type (e.g. secondary spontaneous pneumothorax, catamenial pneumothorax, iatrogenic pneumothorax) were excluded from this study.

The pneumothorax size was assessed using the regression formula derived from Collins et al. [13]. The volume of the resected parietal pleura was measured in cubic centimeter $\left(\mathrm{cm}^{3}\right)$ as noted in the pathology results. The operation time (minutes) was defined as the time from incision to the end of skin closure. A postoperative prolonged air leak was defined as a persistent air leakage for more than 5 days. Recurrence was described as pneumothorax detected on a chest radiograph at presentation in

Table 1 Clinical characteristics

\begin{tabular}{|c|c|c|c|}
\hline Variables & Two-port VATS N $=28$ & Three-port VATS N $=30$ & $p$-value \\
\hline Age (years) & 23 (range 18-40) & 22 (range 18-39) & 0.49 \\
\hline $\operatorname{Sex}(n ; \%)$ & & & 0.97 \\
\hline Female & $5(17.9 \%)$ & $5(16.7)$ & \\
\hline Male & $23(82.1 \%)$ & $25(83.3 \%)$ & \\
\hline Weight (kg) & 70 & 63 & 0.53 \\
\hline Height (m) & 1.80 & 1.80 & 0.77 \\
\hline $\mathrm{BMI}\left(\mathrm{kg} / \mathrm{m}^{2}\right)$ & 21.35 & 20.15 & 0.51 \\
\hline Side of pneumothorax & & & 0.54 \\
\hline Right & $22(78.6 \%)$ & 15 (50\%) & \\
\hline Left & $6(21.4 \%)$ & $15(50 \%)$ & \\
\hline Collins $(A+B+C)(\mathrm{cm})$ & 8.99 & 9.4 & 0.95 \\
\hline Active smoker & & & 0.445 \\
\hline Yes & $7(25 \%)$ & $5(16.7)$ & \\
\hline No & $21(75 \%)$ & $25(83.3 \%)$ & \\
\hline History of pneumothorax & 1 & 3 & 0.336 \\
\hline
\end{tabular}

Unless otherwise specified, all data are presented as mean value. $B M I$ Body-Mass-Index, $\mathrm{cm}$ centimetre, $K g$ kilogram, $m$ metre, A ${ }^{*} p$-value $<0.05$ indicates statistical significance. Collins $(A+B+C)=$ sum of the intrapleural distances $(\mathrm{cm})$ according to the regression formula derived from Collins et al. (13) 
Table 2 Surgical characteristics

\begin{tabular}{|c|c|c|c|}
\hline Variables & $\begin{array}{l}\text { Two-port } \\
\text { VATS } N=28\end{array}$ & $\begin{array}{l}\text { Three-port } \\
\text { VATS N }=30\end{array}$ & $p$-value \\
\hline Chest tube duration (days) & 5,5 & 7 & 0.228 \\
\hline LOS (days) & 7 & 9 & $0.012^{*}$ \\
\hline Piritramide dosage / stay (mg) & 15 & 30 & $0.012^{*}$ \\
\hline Non-opioid dosage / stay (g) & 16 & 20 & $0.010^{*}$ \\
\hline Operation time (min) & 65 & 90 & $0.001 *$ \\
\hline Length of air leak (days) & 5 & 6 & 0.135 \\
\hline Pleurectomy volume $\left(\mathrm{cm}^{3}\right)$ & 12.8 & 14.3 & 0.450 \\
\hline \multicolumn{4}{|l|}{ Postoperative complications } \\
\hline Hemothorax (n, \%) & 0 & $3(10 \%)$ & $0.001^{*}$ \\
\hline Prolonged air leak (n, \%) & $9(32.1 \%)$ & $15(50.0 \%)$ & 0.136 \\
\hline Recurrence $(n, \%)$ & $2(7.1 \%)$ & $5(16.7 \%)$ & 0.274 \\
\hline
\end{tabular}

Unless otherwise specified, all data are presented as mean value. $\mathrm{cm}^{3}$ cubic centimetre, $\min$ minutes, $m g$ milligram, $g$ gram, LOS length of hospital stay. A ${ }^{*} p$-value $<0.05$ indicates statistical significance

our emergency room after surgical treatment by VATS or chest tube drainage. Our standard postoperative medication regime of analgesia (non-opioid) was administered intravenously or orally. The patients received either Metamizol-Natrium $1000 \mathrm{mg}$, Paracetamol $1000 \mathrm{mg}$ or Ibuprofen $600 \mathrm{mg}$ four times per day. In case of persistent pain using the standard pain medication regime, we applied Piritramide (opioid) $7.5 \mathrm{mg}$ intravenously every 4-6 h on patient request. For each patient, the total opioid- and non-opioid dose per stay was evaluated and documented.

One week after discharge from the hospital, the patients visited our outpatient clinic for postoperative control and follow-up. These visits continued in 3 months intervals for one year. For long-term follow-up, the patients were contacted (by telephone call or mail) and assed with a questionnaire. The median follow-up period was 61.6 (range 5-119) months.

The local Institutional Review Board of the HeinrichHeine University Hospital of Duesseldorf approved this study (study Nr: 2020-1271).

\section{Surgical procedures}

Excluding the amount of surgical ports, all patients received the same surgical treatment, consisting of partial pleurectomy and bullectomy when blebs were evident. All operations were performed under general anaesthesia and one lung-ventilation. The patients were placed in a lateral position and the table flexed up to $35^{\circ}$ to open up the intercostal spaces.

\section{Three-Port VATS (Fig. 1)}

For 3-P-VATS, the first $1.5 \mathrm{~cm}$ skin incision was performed at the level of the $5^{\text {th }}$ intercostal space in the

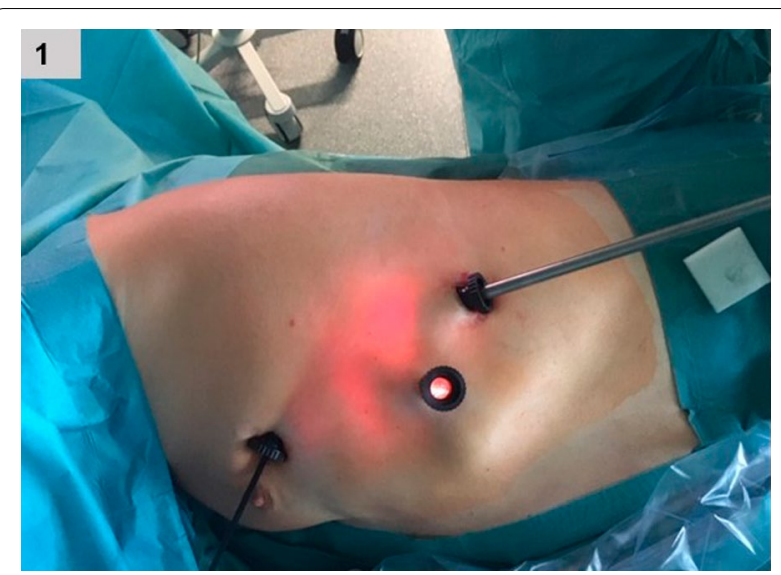

Fig. 1 Trocar position during three-port VATS

anterior axillary line. After placement of an $11 \mathrm{~mm}$ trocar and insertion of the video-thoracoscope, explorative thoracoscopy was performed for thorough inspection of the visceral and parietal pleura. If the patient already had a chest tube, this chest thoracostomy wound (mostly 5 th or 6 th intercostal space of the mid-axillary line) was used for the $11 \mathrm{~mm}$ optical trocar. Under thoracoscopic control, two additional $11 \mathrm{~mm}$ trocars were placed at the level of the 7th and 8th intercostal space in the mid- and posterior-axillary line, respectively (Fig. 1). In case of bullae or blebs, the video-thoracoscope was removed and reinserted through the trocar in the 8th intercostal space. Hereafter, an endograsper and an endoscopic stapling device (Autosuture GIA Universal; Covidien, Mansfield, MA, USA) were inserted through the trocars in the 5th and 7 th intercostal space, respectively. After grasping the bullous area, bullectomy was undertaken with the stapling device. Partial pleurectomy was performed from the apex of the pleura cavity up to the 7th or 8th intercostal space in a blunt manner using an endograsper and a blunt dissector (Endo Peanut ${ }^{\mathrm{TM}}$ Auto suture ${ }^{\mathrm{TM}}$, COVIDIEN ${ }^{\circledR}$, Willow Lane, Mokena, IL), while sparing the regions of the subclavian and internal mammary vessels to avoid damage of these structures.

\section{Two-Port VATS (Fig. 2)}

Two $1.5 \mathrm{~cm}$ skin incisions were made at the level of the $5^{\text {th }}$ and $8^{\text {th }}$ intercostal space in the mid- and anterior axillary line, respectively (Fig. 2). Thoracoscopy and pleurectomy were performed as described in 3-P-VATS. For bullectomy, an endograsper was inserted without trocarguidance through the skin incision in the 5th intercostal space, adjacent to the trocar of endoscopic stapling device. The video-thoracoscope was introduced through the trocar in the 8th intercostal space. 


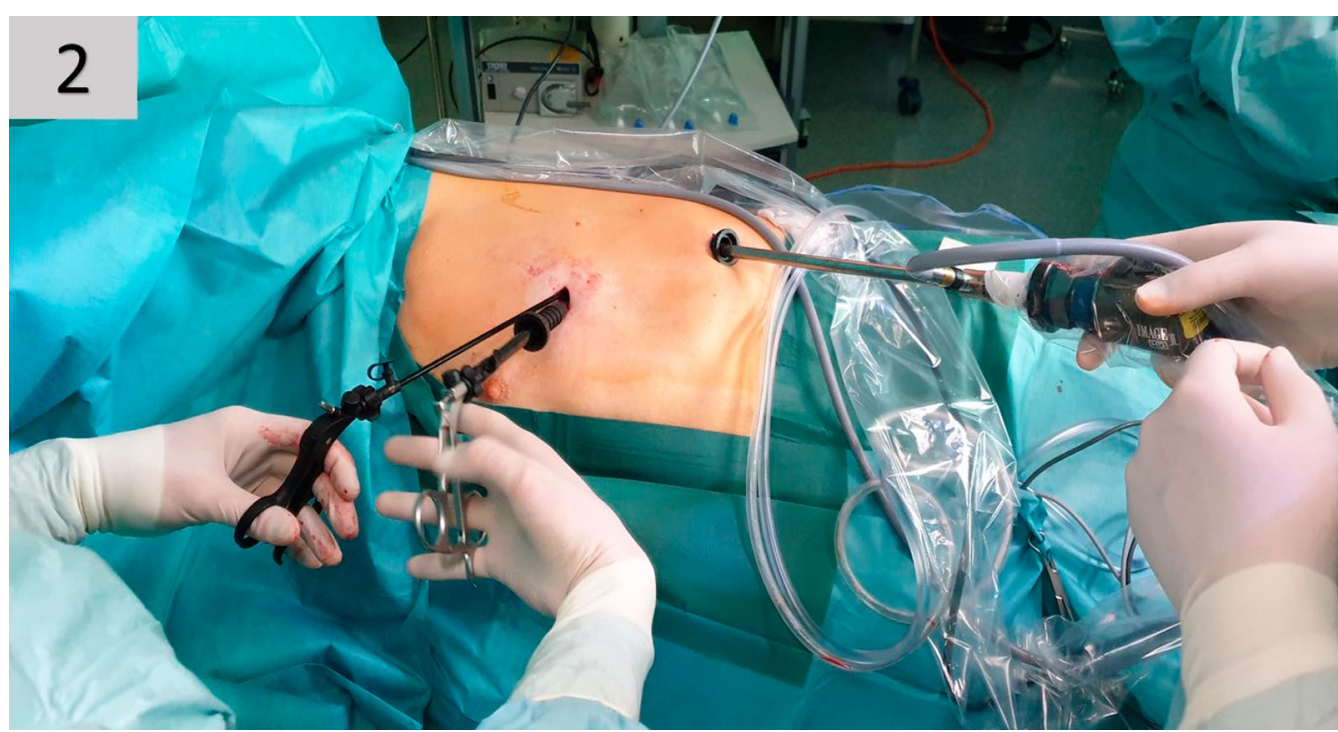

Fig. 2 Trocar placement for two-port VATS

In both procedures, an underwater air leak test was performed. Two $24 \mathrm{Fr}$ chest tubes, placed at the apex of the thorax cavity and in the costodiaphragmatic recess, were inserted through the incisions in the 5th and 8th intercostal spaces, respectively, and connected to a digital chest drainage system (Thopaz +, Medela AG, Baar, Switzerland) with a suction equivalent to $-20 \mathrm{~cm} \mathrm{H}_{2} \mathrm{O}$. During postoperative care, the chest tube drains were removed when no clinical signs of air leak and a drain output less than $200 \mathrm{ml}$ after $24 \mathrm{~h}$ were evident. After chest tube removal, a chest radiograph was taken to verify full expansion of the lung.

Figure 3 shows a summary of the methodological approach of this study.

\section{Statistical analysis}

All data were analysed with the SPSS 25.0 software program (Statistical Package for Social Sciences; SPSS Inc., Chicago, IL, USA). Categorical variables were expressed as percentages and continuous variables were presented as mean. The means of categorical variables were compared by chi-square test or Fisher's exact test, and continuous variables were compared by Student's $t$-test. Statistical significance was considered at $p<0.05$.

\section{Results}

Fifty-eight PSP patients were included in this study. The mean age of the patients was 25 years (range 18-48). Overall, there were 49 male and 9 female patients included. Thirty patients underwent 3-P-VATS, whereas 28 patients received 2-P-VATS. The mean age, sex, side of pneumothorax, smoking behaviour, BMI and pneumothorax size were similar in the 2-P-VATS group and the 3-P-VATS group. The clinical characteristics of the patients are summarised in Table 1.

Surgical characteristics of the two groups are listed in Table 2. There was no significant difference in the number of resected lung segments between both groups, suggesting a lack of selection bias based on the resected lung segments The mean operation time for the 2-Port-VATS was significantly shorter compared with the 3-P-VATS (65 $\mathrm{min}$ vs. $90 \mathrm{~min}, p=0.017$ ). Furthermore, patients operated using 3-P-VATS required a significantly higher total dose of opioid and non-opioid analgesics per stay, compared with patients treated by 2- P-VATS (opioid: $30 \mathrm{mg}$ vs. $15 \mathrm{mg} ; p=0.021$; non-opioid: $16 \mathrm{~g}$ vs. $20 \mathrm{~g}$; $p=0.010)$. 2- P-VATS patients had a significantly shorter LOS compared with 3-P-VATS patients (7 days vs. 9 days; $p=0.012$ ). The duration of chest tube was longer in the 3-P-VATS group compared with the 2-P-VATS group, although this difference did not reach statistical significance (5.5 days vs. 7 days; $p=0.228$ ). Three patients in the 3-P VATS group suffered a postoperative haemothorax, while none of the patients in the 2-P VATS group was affected. Although we observed no significant difference, the pleurectomy volume was larger in the 3-P-VATS patients compared to the patients who underwent 2-P-VATS. One of the patients with postoperative hemothorax required recurrent VATS, the other two patients were successfully treated conservatively. All patients with prolonged air leak received conservative treatment until full recovery. During a median follow-up period of 61.6 months, 5 (16.7\%) patients in the 3-P-VATS group suffered a recurrence, whereas only 2 (7.1\%) patients 


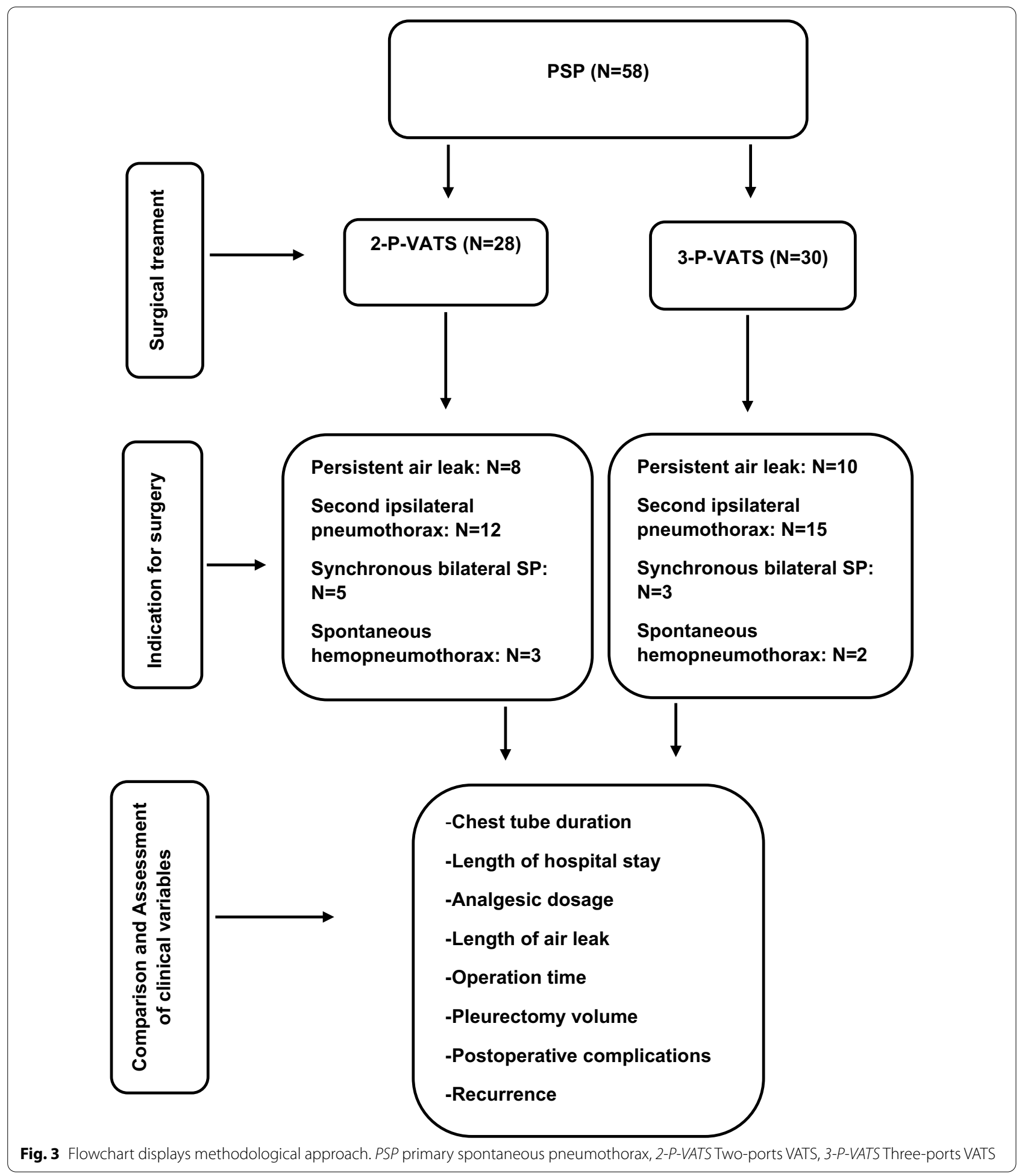

in the 2-P-VATS group experienced recurrence. However, this difference did not reach statistical significance $(p=0.274)$.

\section{Discussion}

In the last decades, thoracic surgery has evolved from thoracotomies to video assisted thoracoscopic surgery (VATS) as the gold standard. While three port VATS still remains the standard-of-care in most centres due to the 
accessibility, recent technical developments are leading to a reduction in access ports. While this may improve postoperative performance such as reduced paraesthesia, analgesic use and LOS [6-11], the more limited access may reduce the operative results. While single-port VATS has been heralded as new minimal access VATS in selected indications and specialized centres, two- and three-port VATS remains the gold standard in most settings. Yet, comparisons of 3-P-VATS with 2-P-VATS have been rarely reported and the impact on postoperative performance as well as effectiveness of the surgical therapy remain elusive.

In the study of Lin F et al. [12] with 23 PSP patients who underwent 2-P-VATS and 73 patients with 3-P-VATS, mean operation time, average LOS and average postoperative chest tube duration were not significantly different between both groups. Similar to our results, postoperative pain was significantly lower in the 2-P-VATS group compared with the 3-P-VATS group. In another recent study of Kutluk AC et al. [8] including 45 patients operated by 2 -P-VATS und 45 patients by 3 -P-VATS, no significant difference was observed in mean operation time, LOS, duration of chest tube drainage, recurrence rates and postoperative pain.

In our study, 28 patients underwent 2-P-VATS and 30 patients received 3-P-VATS. In contrast to the above studies $[8,12]$, we observed a significant difference in operation time, LOS and postoperative dose of analgesic between both groups. Patients operated by 2-P-VATS had a significantly reduced LOS, less postoperative pain and shorter operation time compared with patients operated by 3-P-VATS. In terms of postoperative complications, we observed a significantly higher rate of hemothorax in the 3-P-VATS group. Although not reaching statistical significance, the larger volume of resected parietal pleura in the 3-P-VATS groups $\left(14.3\right.$ vs. $\left.12.8 \mathrm{~cm}^{3}\right)$, as well as the additional port access, may have contributed to the higher rate of hemothorax in the 3-P-VATS group. As described in previous studies, VATS with additional pleurectomy is associated with reduced recurrence rates [14-16]. However, the volume of pleurectomy seems to affect postoperative outcome in terms of complications and postoperative pain. Regarding our study groups, patients who underwent 3-P-VATS suffered a high complications rate and had a high analgesic use compared to patients after 2-P-VATS. We attributed these results to the high pleurectomy volume and the additional port access implemented during 3-P-VATS.

To assess the rate of recurrence, all patients were followed-up for a mean period of 61.6 months. During this period, $5(16.7 \%)$ and $2(7.1 \%)$ patients from the 3-P and 2-P VATS group, respectively, suffered a recurrence. Despite the lower volume of resected pleura in the
2-P-VATS group, there was no significant difference in terms of recurrence rates between both groups.

In previous studies [6-12], postoperative pain was assessed using the visual analogue scale (VAS), which is a subjective measure for pain. In our study, we used the cumulative postoperative dosage of opioid and nonopioid analgesics per patient as an objective surrogate for postoperative pain. Unlike the VAS score, which may give a one-time measurement including the patient's emotional and psychological state, the quantification of applied analgesics allows to assess average pain levels over a longer period. We found a significantly reduced opioid and non-opioid dosage in the 2-P-VATS group, most likely due to the reduced port access. To our knowledge, our study is the first study that elucidates painrelated analgesic use after VATS for patients with PSP.

Our results demonstrate that 2-P-VATS in the treatment of PSP leads to a better postoperative outcome and earlier recovery compared with the conventional 3-P-VATS.

As a retrospect analysis of a limited cohort, this study carries the limitations inherent to this approach. Due to the study period, not all operations were performed by the same surgeon. Nevertheless, our findings, especially the significantly higher rate of postoperative hemothorax observed in our cohort in the 3-P-VATS group, should be further elucidated in larger, randomized controlled trials.

\section{Conclusion}

Our data demonstrate that 2-P-VATS is safer and as effective as 3-P-VATS in the treatment of PSP. It is associated with decreased postoperative pain, reduced length of hospital stay and fewer postoperative complications, indicating that 2-P-VATS should be considered standardof-care in the treatment of PSP.

\section{Abbreviations}

PSP: Primary spontaneous pneumothorax; VAS: Visual analog scale; VATS: Video-assisted thoracoscopic surgery; 3-P-VATS: Three-port VATS; 2-P-VATS: Two-port VATS; LOS: Length of hospital stay; PV: Pleurectomy volume; BMI: Body mass index.

\section{Acknowledgements}

None.

\section{Authors' contributions}

Study conception, design and overall analysis and interpretation of data, drafting, revising the manuscript (SF, HA, SS, AS, AR, LD, GF, WTK), Data analysis and interpretation (SF, HA, AS, LD, GF, WTK) manuscript preparation (SF, GF), conceptual contributions and manuscript revision (SF, HA, SS, LD, AR, GF, WTK). All authors read and approved the final manuscript.

\section{Funding}

Open Access funding enabled and organized by Projekt DEAL. There is no funding or grant support for this manuscript. 


\section{Availability of data and materials}

The datasets used and/or analysed during the current study are available from the corresponding author on reasonable request.

\section{Declarations}

Ethics approval and consent to participate

This study was approved by the local institutional review board (Heinrich Heine University, Duesseldorf, Germany; study-no:: 2020-1271). All procedures performed in this study were in accordance with the ethical standards in the 1964 Declaration of Helsinki and its later amendments. Informed consent to participate was waived because no data regarding the cases were disclosed.

\section{Consent for publication}

Not applicable.

\section{Competing interests}

Drs. Stephen Fung, Hany Ashmawy, Sami Safi M.D, Anja Schauer, Alexander Rehders, Levent Dizdar, Georg Fluegen and Wolfram Trudo Knoefel have no conflicts of interest or financial ties to disclose.

Received: 6 June 2021 Accepted: 8 December 2021

Published online: 18 December 2021

\section{References}

1. Schnell J, Beer M, Eggeling S, Gesierich W, Gottlieb J, Herth FJF, Hofmann HS, Jany B, Kreuter M, Ley-Zaporozhan J, et al. Management of spontaneous pneumothorax and post-interventional pneumothorax: German S3 guideline. Respiration. 2019;97(4):370-402.

2. Sahn SA, Heffner JE. Spontaneous pneumothorax. N Engl J Med. 2000;342(12):868-74.

3. Noppen M. Management of primary spontaneous pneumothorax. Curr Opin Pulm Med. 2003;9(4):272-5.

4. MacDuff A, Arnold A, Harvey J. Management of spontaneous pneumothorax: British Thoracic Society Pleural Disease Guideline 2010. Thorax. 2010. https://doi.org/10.1136/thx.2010.136986.

5. Tschopp JM, Bintcliffe O, Astoul P, Canalis E, Driesen P, Janssen J, Krasnik M, Maskell N, Van Schil P, Tonia T, et al. ERS task force statement: diagnosis and treatment of primary spontaneous pneumothorax. Eur Respir $\mathrm{J}$. 2015:46(2):321-35

6. Salati M, Brunelli A, Xiumè F, Refai M, Sciarra V, Soccetti A, Sabbatini A. Uniportal video-assisted thoracic surgery for primary spontaneous pneumothorax: clinical and economic analysis in comparison to the traditional approach. Interact Cardiovasc Thorac Surg. 2008;7(1):63-6.

7. Jutley RS, Khalil MW, Rocco G. Uniportal vs standard three-port VATS technique for spontaneous pneumothorax: comparison of post-operative pain and residual paraesthesia. Eur J Cardiothorac Surg. 2005;28(1):43-6.

8. Kutluk AC, Kocaturk Cl, Akin H, Erdogan S, Bilen S, Karapinar K, Sezen CB, Saydam $\mathrm{O}$. Which is the best minimal invasive approach for the treatment of spontaneous pneumothorax? Uniport, two, or three ports: a prospective randomized trail. Thorac Cardiovasc Surg. 2018;66(7):589-94.

9. Jeon HW, Kim YD. Does $11.5 \mathrm{~mm}$ guided single port surgery has clinical advantage than multi-port thoracoscopic surgery in spontaneous pneumothorax? J Thorac Dis. 2016;8(10):2924-30.

10. Tamura M, Shimizu Y, Hashizume Y. Pain following thoracoscopic surgery: retrospective analysis between single-incision and three-port videoassisted thoracoscopic surgery. J Cardiothorac Surg. 2013:8:153.

11. Kang DK, Min HK, Jun HJ, Hwang YH, Kang MK. Early outcomes of singleport video-assisted thoracic surgery for primary spontaneous pneumothorax. Korean J Thorac Cardiovasc Surg. 2014;47(4):384-8.

12. Lin F, Zhang C, Zhang Q, Dang D, Zhao Y. Postoperative pain in treatment of spontaneous pneumothorax with limited two-port thoracoscopy. Pak J Med Sci. 2014;30(4):712-5.

13. Collins CD, Lopez A, Mathie A, Wood V, Jackson JE, Roddie ME. Quantification of pneumothorax size on chest radiographs using interpleural distances: regression analysis based on volume measurements from helical CT. AJR Am J Roentgenol. 1995;165(5):1127-30.
14. Mithiran H, Leow L, Ong K, Liew T, Siva D, Liang S, Tam JKC. Video-assisted thoracic surgery (VATS) talc pleurodesis versus pleurectomy for primary spontaneous pneumothorax: a large single-centre study with no conversion. World J Surg. 2019;43(8):2099-105.

15. Neudecker J, Malzahn U, Heuschmann P, Behrens U, Walles T. Pulmonary wedge resection plus parietal pleurectomy (WRPP) versus parietal pleurectomy (PP) for the treatment of recurrent primary pneumothorax (WOPP trial): study protocol for a randomized controlled trial. Trials. 2015;16:540.

16. Shaikhrezai K, Thompson Al, Parkin C, Stamenkovic S, Walker WS. Videoassisted thoracoscopic surgery management of spontaneous pneumothorax-long-term results. Eur J Cardiothorac Surg. 2011;40(1):120-3.

\section{Publisher's Note}

Springer Nature remains neutral with regard to jurisdictional claims in published maps and institutional affiliations.
Ready to submit your research? Choose BMC and benefit from:

- fast, convenient online submission

- thorough peer review by experienced researchers in your field

- rapid publication on acceptance

- support for research data, including large and complex data types

- gold Open Access which fosters wider collaboration and increased citations

- maximum visibility for your research: over $100 \mathrm{M}$ website views per year

At BMC, research is always in progress.

Learn more biomedcentral.com/submissions 